\title{
A convenient access to 1-substituted-2-azinyl-1-ethanones via acylation of alkylated azines with $N$-acylbenzotriazoles
}

\author{
Alan R. Katritzky,* Ashraf A. A. Abdel-Fattah, and Rena G. Akhmedova \\ Center for Heterocyclic Compounds, Department of Chemistry, University of Florida, \\ Gainesville, FL 32611-7200 \\ E-mail: Katritzky@chem.ufl.edu
}

\begin{abstract}
Submitted in Honor of the $55^{\text {th }}$ anniversary of Professor Eusebio Juaristi (received 15 Jun 05; accepted 12 Aug 05; published on the web 17 Aug 05)
\end{abstract}

\begin{abstract}
Reactions of alkylazines 9a-f (2-methylpyridine, 2-benzylpyridine, 4-benzylpyridine, 2-methylquinoline, 4-methylquinoline or 4-methylpyrimidine) with readily available $N$-acylbenzotriazoles $\mathbf{8 a}-\mathbf{j}$ produced 1-substituted-2-azinyl-1-ethanones 10a-p in 50-95\% yields.
\end{abstract}

Keywords: $N$-Acylbenzotriazoles, alkylated azines, 1-substituted-2-azinyl-1-ethanones

\section{Introduction}

1,2-Disubstituted-1-ethanones ( $\left.\mathrm{X}-\mathrm{CH}_{2} \mathrm{CO}-\mathrm{Y}\right)$ play an important role in organic synthesis. ${ }^{1}$ Among such derivatives, 1-substituted-2-azin-2-yl-1-ethanones ( $\left.\mathrm{Het}-\mathrm{CH}_{2} \mathrm{COY}\right)$ find important and widespread uses as ligands to chelate transition metals ${ }^{2}$ and medically important gallium ${ }^{66} \mathrm{Ga},{ }^{67} \mathrm{Ga}$, and ${ }^{68} \mathrm{Ga}$ radioisotopes. ${ }^{3}$ Compounds of this class are also useful as synthetic templates in the preparation of chiral building blocks ${ }^{4}$ for a wide variety of alkaloids, e.g., hydrangea $1,{ }^{5}$ lamellarins $2{ }^{6}$ sedamines $3^{7}$ and lobelines 4 and $5^{8}$ as well as steroid-like compounds such as azasteroids 6. ${ }^{9}$ These systems have long been recognized as bioactive natural products. ${ }^{10}$ For example, early findings on the biological properties of lobelines (also known as Indian tobacco) support their potential ability to exhibit agonist activity ${ }^{8}$ and enhance latent inhibition $^{11}$ at nicotinic receptors, stimulate autonomic ganglia, ${ }^{12}$ and improve memory. ${ }^{10 a}$

Moreover, certain 1-substituted-2-azinyl-1-ethanone derivatives exhibit biological activities as potential hypocholesteremic agents having minimal estrogenic activity ${ }^{13}$ and others are valuable synthons in the development of various pharmaceutically important molecules ${ }^{1 \mathrm{a}, 14}$ and in the preparation of chiral nematic materials. ${ }^{15}$

Previous protocols for syntheses of 1-substituted-2-azinyl-1-ethanones (Scheme 1) include: (i) from 1-substituted-2-azinylethynes by hydration in $2 \mathrm{~N} \mathrm{H}_{2} \mathrm{SO}_{4}$ in the presence of $\mathrm{HgCl}_{2} ;{ }^{16}$ (ii) reactions of organometallic reagents with aldehyde followed by Swern oxidation; ${ }^{17}$ (iii) radical 
nucleophilic substitution reactions $\left(\mathrm{S}_{\mathrm{RN}} 1\right)$ of haloazines with ketone enolates; ${ }^{18}$ (iv) reactions of $\alpha$-haloazinium salts (pseudo-Vilsmeier reagents) with $N, N$-disubstituted enamines followed by acidic hydrolysis and dequaternization; ${ }^{19}$ and $(\mathrm{v})$ acylation of methylated azines.

Approaches of type (v) are the most commonly used and include treatment of methlyazines with carbonitriles $^{20}$ or with activated derivatives of carboxylic acids, especially acid chlorides, ${ }^{21}$ esters, $^{13,22}$ and amides. ${ }^{23}$<smiles>O=C(CC1NCCCC1O)Cn1cnc2ccccc2c1=O</smiles>

1<smiles>[R]C(=O)CC1CC=CCN1[R]</smiles>

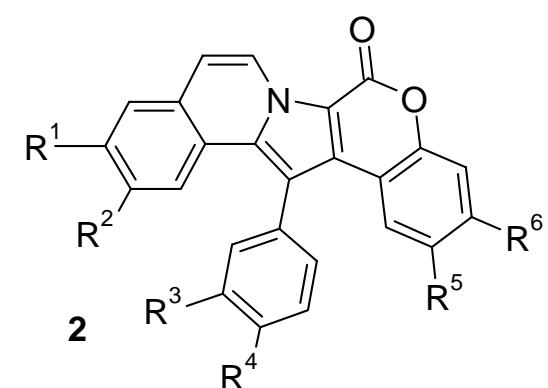<smiles>C[C@]12CC[C@@H](O)C[C@H]1CCN1CCCC[C@@H]12</smiles><smiles>[R]C(O)CC1CCCCN1C</smiles>

6

\section{Figure 1}

Claisen-type condensation methods in refs $^{22 a-c}$ require either a $2: 1$ molar ratio of metallated alkylazine:acylating ester or 2 molar equivalents of base that might cause ester selfcondensation prior to the lateral acylation. Among the conventional methods available for the synthesis of 1-substituted-2-azinyl-1-ethanones, approaches based on the use of amides as acylating reagents are scarce.

We have reported earlier the use of $N$-acylbenzotriazoles in the syntheses of amides, ${ }^{24}$ esters, ${ }^{25} \beta$-keto sulfones ${ }^{26}$ and $\beta$-keto nitriles. ${ }^{27}$ In view of our previous results, we now describe a further application of $N$-acylbenzotriazoles in a general and convenient access to a variety of 1substituted-2-azinyl-1-ethanones in satisfactory to excellent yields by the acylation of alkylated azines.

\section{Results and Discussion}

The starting $N$-acylbenzotriazoles 8a-f with alkyl, alkenyl or aryl substituents $(\mathrm{R}=$ isobutyl, $n$ butyl, cinnamyl, phenyl, 4-chlorophenyl or 4-nitrophenyl) were prepared by stirring acid chlorides in $\mathrm{CH}_{2} \mathrm{Cl}_{2}$ with benzotriazole in the presence of $\mathrm{Et}_{3} \mathrm{~N}$ at room temperature ${ }^{28}$ while $\mathbf{8 g}-\mathbf{j}$ ( $\mathrm{R}=$ 4-diethylaminophenyl, 3-pyridyl, 2-thienyl or 2-furyl) were readily prepared by refluxing 
the corresponding carboxylic acids in THF with 1-(methansulfonyl)-1H-benzotriazole in the presence of $\mathrm{Et}_{3} \mathrm{~N}$. $^{24 \mathrm{a}}$

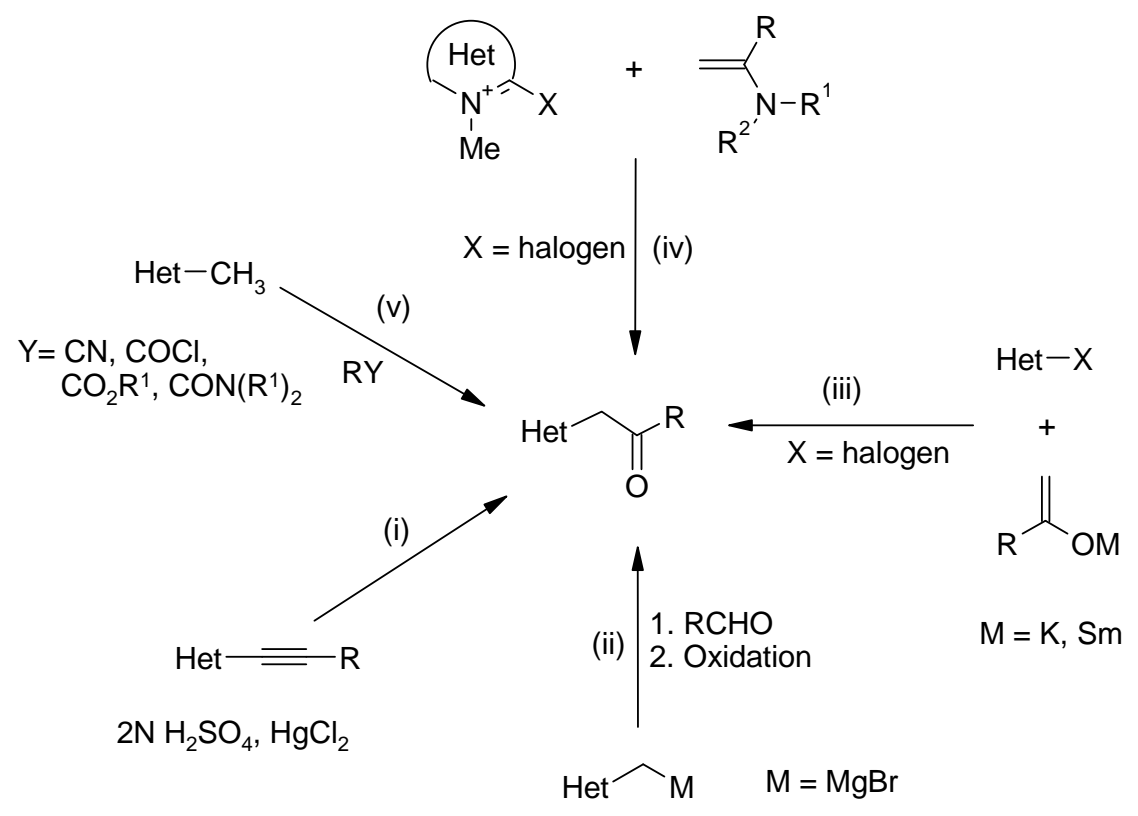

\section{Scheme 1}

The acylation reactions were accomplished by treatment of alkylazines 9a-f (1.0 equivalent) in THF at $-78{ }^{\circ} \mathrm{C}$ with LDA (2.0 equivalents), itself prepared in situ from $n$ butyllithium and diisopropylamine), followed by the addition, at $-78{ }^{\circ} \mathrm{C}$, of a THF solution of the appropriate $N$-acylbenzotriazole $\mathbf{8 a}-\mathbf{j}$ (1.0 equivalent). The solution was allowed to warm up to room temperature overnight. After aqueous workup, 1-substituted-2-azinyl-1-ethanones 10a-p were isolated as the only products in good to excellent yields (Scheme 2, Table 1). This approach provided known compounds $\mathbf{1 0 a}, \mathbf{d}, \mathbf{i}^{22 \mathrm{a}}$ and $\mathbf{1 0 b ^ { 2 9 }}$ in yields comparable with those reported in the literature and dramatically improved the previous yields of $\mathbf{1 0 f}, \mathbf{1 0 g}, \mathbf{1 0 h}$, and $\mathbf{1 0 j}$ from $12 \%,{ }^{13}$ $41 \%{ }^{22 \mathrm{~d}} 58 \%{ }^{22 \mathrm{a}}$ and $50.3 \%^{22 \mathrm{~b}}$ to $91 \%, 95 \%, 84 \%$ and $72 \%$ yield, respectively.

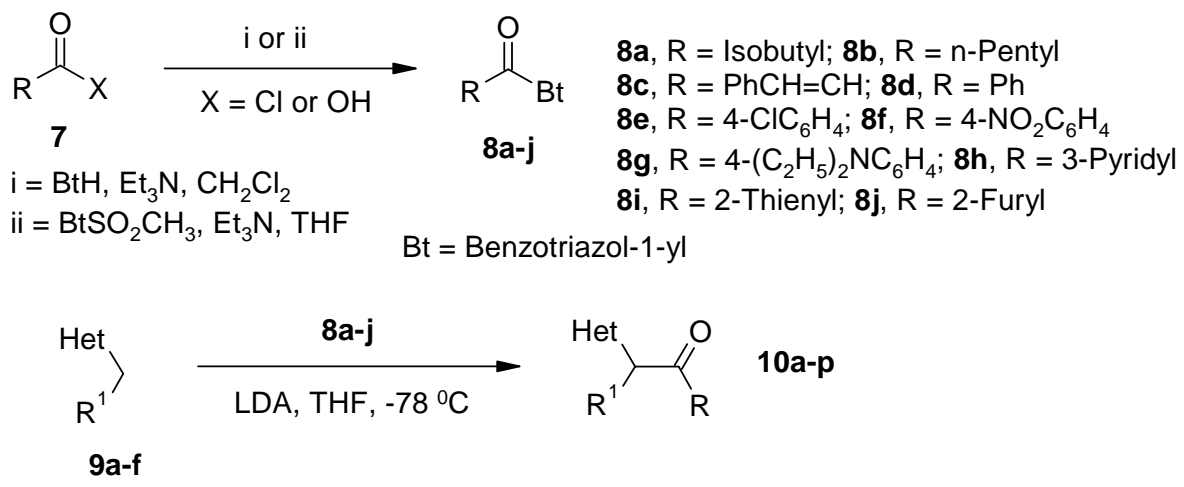

\section{Scheme 2}

For designation of $\mathrm{R}, \mathrm{R}^{1}$ and Het in $\mathbf{1 0}$ see Table 1 
Table 1. Preparation of 1-Substituted-2-azinyl-1-ethanones 10a-p

\begin{tabular}{|c|c|c|c|c|c|}
\hline Entry & Het & $\begin{array}{l}\mathrm{R} \text { of } \\
\mathrm{RCOBt} \mathbf{8}\end{array}$ & $\begin{array}{l}\mathrm{R}^{1} \text { of } \\
\mathrm{HetCH}_{2} \mathrm{R}^{1} \mathbf{9}\end{array}$ & $\begin{array}{l}\text { Yield }(\%)^{\mathrm{a}} \\
(\text { keto + enol) }\end{array}$ & $\begin{array}{c}\text { Keto/ enol } \\
(\%)^{b}\end{array}$ \\
\hline 10a & Pyrid-2-yl & $\left(\mathrm{CH}_{3}\right)_{2} \mathrm{CHCH}_{2}$ & $\mathrm{H}$ & $65\left(60^{22 a}\right)^{c}$ & $95 / 5$ \\
\hline 10b & Pyrid-2-yl & $\mathrm{CH}_{3}\left(\mathrm{CH}_{2}\right)_{3} \mathrm{CH}_{2}$ & $\mathrm{H}$ & $60\left(52^{29}\right)^{c}$ & $100 / 0$ \\
\hline 10c & Pyrid-2-yl & $\mathrm{PhCH}=\mathrm{CH}$ & $\mathrm{H}$ & 65 & $27 / 73$ \\
\hline 10d & Pyrid-2-yl & $\mathrm{Ph}$ & $\mathrm{H}$ & $78\left(82^{22 a}\right)^{c}$ & $59 / 41$ \\
\hline $10 \mathrm{e}$ & Pyrid-2-yl & 4- $\mathrm{ClC}_{6} \mathrm{H}_{4}$ & $\mathrm{H}$ & 83 & $38 / 62$ \\
\hline $10 f$ & Pyrid-2-yl & $4-\mathrm{ClC}_{6} \mathrm{H}_{4}$ & $\mathrm{Ph}$ & $95\left(12^{13}\right)^{\mathrm{c}}$ & $58 / 42$ \\
\hline $10 \mathrm{~g}$ & Quinolin-2-yl & $\mathrm{Ph}$ & $\mathrm{H}$ & $91\left(41^{22 d}\right)^{c}$ & 8/ 92 \\
\hline $10 \mathrm{~h}$ & Pyrid-2-yl & Fur-2-yl & $\mathrm{H}$ & $84\left(58^{22 \mathrm{a}}\right)^{\mathrm{c}}$ & $68 / 32$ \\
\hline $10 \mathrm{i}$ & Pyrid-2-yl & Thien-2-yl & $\mathrm{H}$ & $68\left(73^{22 \mathrm{a}}\right)^{\mathrm{c}}$ & $85 / 15$ \\
\hline $10 \mathrm{j}$ & Pyrid-2-yl & Pyrid-3-yl & $\mathrm{H}$ & $72\left(50^{22 b}\right)^{\mathrm{c}}$ & $16 / 84$ \\
\hline $10 \mathrm{k}$ & Pyrimidin-4-yl & Fur-2-yl & $\mathrm{H}$ & 50 & $50 / 50$ \\
\hline 101 & Quinolin-4-yl & Thien-2-yl & $\mathrm{H}$ & 66 & $100 / 0$ \\
\hline $10 m$ & Pyrid-4-yl & $4-\mathrm{ClC}_{6} \mathrm{H}_{4}$ & $\mathrm{Ph}$ & 63 & $100 / 0$ \\
\hline 10n & Pyrid-4-yl & $4-\left(\mathrm{C}_{2} \mathrm{H}_{5}\right)_{2} \mathrm{NC}_{6} \mathrm{H}_{4}$ & $\mathrm{Ph}$ & 67 & $100 / 0$ \\
\hline 100 & Quinolin-4-yl & $4-\mathrm{ClC}_{6} \mathrm{H}_{4}$ & $\mathrm{H}$ & 87 & $100 / 0$ \\
\hline $10 p$ & Quinolin-4-yl & 4- $\mathrm{NO}_{2} \mathrm{C}_{6} \mathrm{H}_{4}$ & $\mathrm{H}$ & 72 & $100 / 0$ \\
\hline
\end{tabular}

${ }^{\mathrm{a}}$ Products were recovered as mixture of keto/enol tautomers as evidenced by ${ }^{1} \mathrm{H} \mathrm{NMR}$ in $\mathrm{CDCl}_{3}$ with the exceptions of $\mathbf{1 0 b}, \mathbf{l}-\mathbf{p}$, where the percentage of the keto form was $100 \%$. ${ }^{\mathrm{b}}$ Determined by ${ }^{1} \mathrm{H}$ NMR of products $10 .{ }^{\mathrm{c}}$ Literature yield.

The structures of the novel condensation products $10 \mathbf{c}, \mathbf{e}, \mathbf{k}-\mathbf{p}$ are supported by their spectroscopic data together with microanalyses and known compounds $\mathbf{1 0 a , b , d , f - j}$ by comparison of their melting points and spectroscopic data with the literature reports together with microanalyses in some cases. In nearly all cases, the acylated products derived from 2alkylazines exist in $\mathrm{CDCl}_{3}$ solution as tautomeric mixtures. Their ${ }^{1} \mathrm{H}$ NMR spectra display two closely overlapping sets of signals and their proton-decoupled ${ }^{13} \mathrm{C}$ NMR spectra generally show two sets of lines. By comparison of the magnitudes of the enolic and ketonic shifts to values from the literature, ${ }^{30}$ they were identified as the ketone and enol forms $\mathbf{K}$ and $\mathbf{O}$; enaminone tautomers $\mathbf{E}$ were not observed (Scheme 3). The integrated intensities of the side-chain methylene and vinyl protons in the ${ }^{1} \mathrm{H}$ NMR spectra of $\mathrm{CDCl}_{3}$ solutions of $\mathbf{1 0 a}-\mathbf{k}$ indicated a predominance of the $\mathbf{O}$ forms in 10c,e,g,j $62-92 \%$ while $\mathbf{K}$ forms predominated in 10a,d,f,h,i 58-95\%. Although ${ }^{1} \mathrm{H}$ NMR spectra showed a tautomeric mixture of about $1: 1$ for $10 \mathbf{k}, 10 \mathbf{b}$ was observed exclusively in the $\mathrm{K}$ form. This finding is in accord with the facts that hydrogenbonding reinforces tautomeric effects ${ }^{31}$ and tautomer ratio is crucially dependent on both steric and polar effects of the substituents attached to the carbonyl group ${ }^{30}$ (Scheme 3). However, the 
condensation products 101-p derived from 4-benzylpyridine and 4-methylquinoline exist predominantly in the ketonic form.

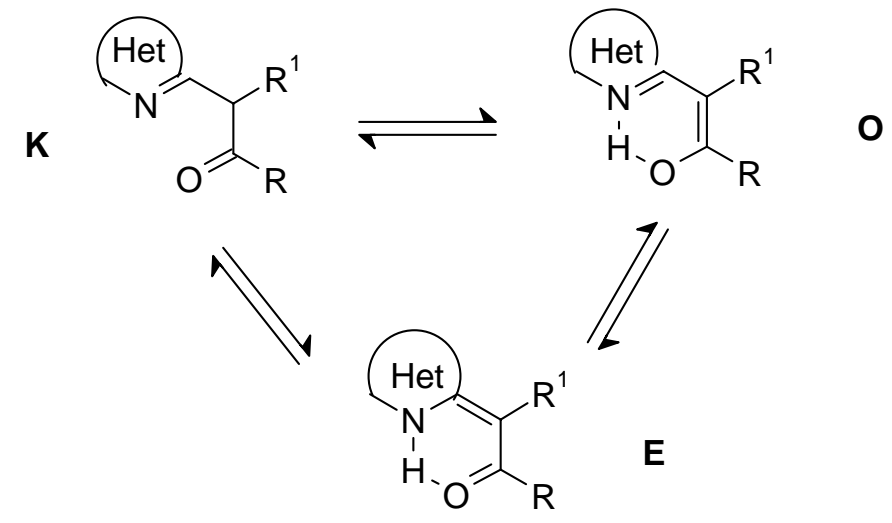

\section{Scheme 3}

This new synthetic procedure for the preparation of 1-substituted-2-azinyl-1-ethanones offers several advantages. Good generality has been demonstrated since a variety of alkyated azines can be acylated with aliphatic (to give 10a,b), alkenyl (to give 10c), benzenoid (to give $\mathbf{1 0 d}-\mathbf{g}, \mathbf{m}-\mathbf{p}$ ) and heterocyclic acylation reagents (to give $\mathbf{1 0 h}-\mathbf{l}$ ). The high average yield for the 8 previously known examples (78\%) compared to the reported $(47 \%)$ and the overall average yield for 16 examples (73\%), confirm once again the superiority of $N$-acylbenzotriazoles as an alternative class of acylating reagents, which extend and complement the arsenal of reagents for acylations of alkylated heterocycles. Compared with acid chlorides, the advantage of $\mathrm{N}$ acylbenzotriazoles resides in their neutral character and high stability. While $N, N-$ dimethylamides $^{23}$ have been used successfully for the synthesis of 1-alkyl-2-pyridin-2-yl-1ethanones, they are not as easily accessible as the corresponding $N$-acylbenzotriazoles.

In summary, we have developed a convenient and quite general method for the synthesis of 1,2-disubstituted-1-ethanones having a pyridyl, quinolyl or pyrimidyl moiety that describes the potentiality of $\mathrm{N}$-acylbenzotriazoles as valuable acylating agents of alkyated azines.

\section{Experimental Section}

General Procedures. All reactions were carried out under an atmosphere of nitrogen, unless otherwise specified. Glassware was routinely oven-dried at $160{ }^{\circ} \mathrm{C}$ for a minimum of $4 \mathrm{~h}$ and then connected to a vacuum line before assembling under a dry argon stream. Anhydrous solvents were obtained by distillation immediately prior to use, from sodium benzophenone ketyl (tetrahydrofuran). Melting points were uncorrected. ${ }^{1} \mathrm{H}$ NMR $(300 \mathrm{MHz})$ and ${ }^{13} \mathrm{C} \mathrm{NMR}(75$ $\mathrm{MHz})$ spectra were recorded using deuteriochloroform $\left(\mathrm{CDCl}_{3}\right)$ as solvent. Column chromatography was carried out on silica gel (230-400 mesh). 


\section{General procedure for the preparation of 1-substituted-2-azinyl-1-ethanones 10a-p}

To a solution of LDA (4.0 mmol) (prepared in situ from diisopropylamine and $n$-butyllithium in THF at $\left.-78{ }^{\circ} \mathrm{C}\right)$, a solution of the alkylated heterocycle $9(2.0 \mathrm{mmol})$ in dry THF $(15 \mathrm{~mL})$ was added dropwise under argon. After stirring the resulting mixture for $1 \mathrm{~h}$ at this temperature to ensure complete carbanion formation, a solution of $N$-acylbenzotriazole $8(2.0 \mathrm{mmol})$ in dry THF $(10 \mathrm{~mL})$ was added dropwise at $-78{ }^{\circ} \mathrm{C}$. The reaction mixture was allowed to warm to $\mathrm{rt}$ overnight before quenching with water $(50 \mathrm{~mL})$ and extraction with EtOAc $(3 \times 30 \mathrm{~mL})$. The combined organic layers were washed with water $(50 \mathrm{~mL})$, dried over $\mathrm{MgSO}_{4}$. The solvent was removed under vacuum and the residue was placed in a silica-gel column and eluted with hexanes/EtOAc 10:1 followed by recrystallization from $\mathrm{CH}_{2} \mathrm{Cl}_{2} /$ hexanes for solid products to give the pure 1-substituted-2-azinyl-1-ethanones 10. All ${ }^{1} \mathrm{H}$ and ${ }^{13} \mathrm{C}$ NMR signals for tautomeric compounds are for [keto + enol] unless otherwise specified.

3-Methyl-1-(pyrid-2-yl)-2-pentanone (10a). Colorless oil ${ }^{22 \mathrm{a}}(65 \%) .{ }^{1} \mathrm{H}$ NMR [keto + enol] $\delta$ $8.54(\mathrm{dd}, J=4.8,1.0 \mathrm{~Hz}, 1 \mathrm{H}), 8.20(\mathrm{br} \mathrm{d}, 5.0 \mathrm{~Hz}, 1 \mathrm{H}), 7.64(\mathrm{dt}, J=7.6,1.8 \mathrm{~Hz}, 1 \mathrm{H}), 7.52(\mathrm{td}, J=$ $7.5,1.5 \mathrm{~Hz}, 1 \mathrm{H}), 7.23-7.15(\mathrm{~m}, 2 \mathrm{H}), 6.92-6.86(\mathrm{~m}, 2 \mathrm{H}), 5.30$ (enol s, 0.05H), 3.92 (keto s, $1.9 \mathrm{H}), 2.47(\mathrm{~d}, \mathrm{~J}=6.7 \mathrm{~Hz}, 2 \mathrm{H}), 2.16$ (septet, $J=6.6 \mathrm{~Hz}, 1 \mathrm{H}), 0.89(\mathrm{~d}, J=6.6 \mathrm{~Hz}, 6 \mathrm{H}) .{ }^{13} \mathrm{C}$ NMR $[$ keto + enol] $\delta 200.9,169.2,158.5,154.7,149.3,144.2,136.7,136.4,124.0,121.7,120.2,117.8$, 95.9, 52.6, 51.4, 45.4, 26.2, 24.2, 22.3 (2C). Anal. Calcd. For $\mathrm{C}_{11} \mathrm{H}_{15} \mathrm{NO}$ : N, 8.48. Found: N, 8.22 .

1-(Pyrid-2-yl)-2-heptanone (10b). Colorless oil ${ }^{29}$ (60\%). ${ }^{1} \mathrm{H}$ NMR $\delta 8.57(\mathrm{~d}, J=4.8 \mathrm{~Hz}, 1 \mathrm{H})$, $7.66(\mathrm{td}, J=7.7,1.8 \mathrm{~Hz}, 1 \mathrm{H}), 7.25-7.18(\mathrm{~m}, 2 \mathrm{H}), 3.94(\mathrm{~s}, 2 \mathrm{H}), 2.53(\mathrm{t}, J=7.4 \mathrm{~Hz}, 2 \mathrm{H})$, $1.34-1.19(\mathrm{~m}, 6 \mathrm{H}), 0.86(\mathrm{t}, J=7.1 \mathrm{~Hz}, 3 \mathrm{H}) .{ }^{13} \mathrm{C}$ NMR $\delta 207.6,154.7,149.2,136.7,124.2,121.9$, 52.0, 42.7, 31.1, 23.3, 22.3, 13.8 .

(E)-4-Phenyl-1-pyrid-2-yl-but-3-en-2-one (10c). Yellow prisms (65\%), mp 90-92 ${ }^{\circ} \mathrm{C} .{ }^{1} \mathrm{H}$ NMR [keto + enol] $\delta 14.6($ enol br s, $0.73 \mathrm{H}), 8.58(\mathrm{~d}, J=8.1 \mathrm{~Hz}, 1 \mathrm{H}), 8.43(\mathrm{~d}, J=8.1 \mathrm{~Hz}, 1 \mathrm{H}), 8.35(\mathrm{~d}$, $J=5.0 \mathrm{~Hz}, 1 \mathrm{H}), 8.16(\mathrm{~d}, J=7.2 \mathrm{~Hz}, 1 \mathrm{H}), 8.15(\mathrm{~s}, 1 \mathrm{H}), 7.78-7.47(\mathrm{~m}, 8 \mathrm{H}), 7.40-7.26(\mathrm{~m}, 5 \mathrm{H})$, $7.05-6.97(\mathrm{~m}, 2 \mathrm{H}), 6.84(\mathrm{~d}, J=16.2 \mathrm{~Hz}, 1 \mathrm{H}), 6.59(\mathrm{~d}, J=15.9 \mathrm{~Hz}, 1 \mathrm{H}), 5.60$ (enol s, 0.73H), 4.18 (keto s, 0.54H). ${ }^{13} \mathrm{C}$ NMR [keto + enol] $\delta$ 190.1, 158.7, 148.8, 145.5, 137.0, 136.6, 132.0, $131.5,130.3,129.1,129.0,128.9,128.7,128.4,128.2,127.1,126.2,124.5,121.6,120.2,119.0$, 116.1, 114,8, 100.6, 100.3, 50.7. HRMS For $\mathrm{C}_{15} \mathrm{H}_{13} \mathrm{NO}: 223.2743$. Found: 223.1006

1-Phenyl-2-(pyrid-2-yl)-1-ethanone (10d). Yellow needles $(78 \%)$, mp $67-69{ }^{\circ} \mathrm{C}$ (lit. $^{22 \mathrm{a}}$ 52.5-54.0 $\left.{ }^{0} \mathrm{C}\right) .{ }^{1} \mathrm{H}$ NMR [ [keto + enol] $\delta 8.56(\mathrm{~d}, J=4.3 \mathrm{~Hz}, 1 \mathrm{H}), 8.28(\mathrm{~d}, J=4.9 \mathrm{~Hz}, 1 \mathrm{H}), 8.07$ $(\mathrm{d}, J=7.1 \mathrm{~Hz}, 2 \mathrm{H}), 7.85(\mathrm{dd}, J=8.0,2.1 \mathrm{~Hz}, 2 \mathrm{H}), 7.66-7.52(\mathrm{~m}, 3 \mathrm{H}) 7.47-7.38(\mathrm{~m}, 4 \mathrm{H}), 7.30$ $(\mathrm{d}, J=7.8 \mathrm{~Hz}, 2 \mathrm{H}), 7.18-7.14(\mathrm{~m}, 1 \mathrm{H}), 7.06(\mathrm{~d}, J=8.1 \mathrm{~Hz}, 1 \mathrm{H}), 6.99-6.95(\mathrm{~m}, 1 \mathrm{H}), 6.8($ enol s, $0.41 \mathrm{H}), 4.50$ (keto s, $1.18 \mathrm{H}$ ). ${ }^{13} \mathrm{C}$ NMR [keto + enol] $\delta 196.8164 .3,158.5,155.2,149.5,144.2$, $138.8,137.1,136.5,136.4,133.2,129.3,128.7,128.6,128.3,125.4,124.2,121.9,121.5,118.4$, 94.1, 48.4. Anal. Calcd. For $\mathrm{C}_{13} \mathrm{H}_{11} \mathrm{NO}$ : C, 79.16; H, 5.62; N, 7.10. Found: C, 79.15; H, 5.63; N, 7.08 .

1-(4-Chlorophenyl)-2-(pyrid-2-yl)-1-ethanone (10e). Yellow needles (83\%), mp 96-97 ${ }^{\circ} \mathrm{C} .{ }^{1} \mathrm{H}$ NMR [keto + enol] $\delta 8.45(\mathrm{~d}, J=4.4 \mathrm{~Hz}, 1 \mathrm{H}), 8.27(\mathrm{~d}, J=4.8 \mathrm{~Hz}, 1 \mathrm{H}), 8.01(\mathrm{~d}, J=8.4 \mathrm{~Hz}, 2 \mathrm{H})$, 
$7.77(\mathrm{~d}, J=8.5 \mathrm{~Hz}, 2 \mathrm{H}), 7.66-7.58(\mathrm{~m}, 2 \mathrm{H}), 7.42(\mathrm{~d}, J=8.6 \mathrm{~Hz}, 2 \mathrm{H}), 7.36(\mathrm{~d}, J=8.4 \mathrm{~Hz}, 2 \mathrm{H})$, 7.29 (d, $J=7.8 \mathrm{~Hz}, 1 \mathrm{H}), 7.19-7.15(\mathrm{~m}, 1 \mathrm{H}), 7.05$ (d, $J=8.1 \mathrm{~Hz}, 1 \mathrm{H}), 7.00-6.98(\mathrm{~m}, 1 \mathrm{H}), 6.03$ (enol s, 0.62H), 4.45 (keto s, 0.76H). ${ }^{13} \mathrm{C}$ NMR [keto + enol] $\delta 195.7,163.5,158.2,154.9,149.6$, $144.0,139.7,137.2$, 136.6, 135.1, 135.0, 130.2, 128.9, 128.5, 126.8, 124.1, 122.0, 121.6, 11.6, 100.2, 94.1, 48.5. Anal. Calcd. For $\mathrm{C}_{13} \mathrm{H}_{10} \mathrm{ClNO}$ : C, 67.40; H, 4.35; N, 6.05. Found: C, 67.38; H, $4.30 ; \mathrm{N}, 5.93$.

1-(4-Chlorophenyl)-2-phenyl-2-(pyrid-2-yl)-1-ethanone (10f). Yellow prisms (95\%), mp 90-92 ${ }^{\circ} \mathrm{C}$ (lit. $\left.{ }^{13} 95-98{ }^{\circ} \mathrm{C}\right) .{ }^{1} \mathrm{H}$ NMR $[$ keto + enol] $\delta 8.54(\mathrm{~d}, J=4.8 \mathrm{~Hz}, 1 \mathrm{H}), 8.34(\mathrm{~d}, J=5.1 \mathrm{~Hz}$, $1 \mathrm{H}), 7.95(\mathrm{~d}, J=8.5 \mathrm{~Hz}, 2 \mathrm{H}), 7.64-7.50(\mathrm{~m}, 2 \mathrm{H}), 7.37-6.99(\mathrm{~m}, 19 \mathrm{H}), 6.84(\mathrm{~d}, J=8.4 \mathrm{~Hz}, 1 \mathrm{H})$, 6.21 (keto s, 0.58H). ${ }^{13} \mathrm{C}$ NMR [keto + enol] $\delta 196.3,163.3,159.8,159.0,149.3,143.0,139.5$, $137.7,137.3,137.2,136.7,136.5,134.9,133.7,132.5,130.4,130.3,129.1,129.0,128.9,128.8$, 127.6, 127.5, 127.0, 123.8, 122.1, 120.8, 118.7, 109.0, 62.1. Anal. Calcd. For $\mathrm{C}_{19} \mathrm{H}_{14} \mathrm{ClNO}$ : C, 74.15; H, 4.58; N, 4.55. Found: C, 74.23; H, 4.56; N, 4.59.

(Z)-1-Phenyl-2-quinolin-2-ylethanol (10g). Yellow needles (91\%), mp $114-116{ }^{\circ} \mathrm{C}$ (lit. $^{22 \mathrm{~d}}$ 115.5-117.0 $\left.{ }^{\circ} \mathrm{C}\right) .{ }^{1} \mathrm{H}$ NMR $\delta 15.78$ (enol br s, 0.92H), $8.02(\mathrm{dd}, J=5.9,2.2 \mathrm{~Hz}, 2 \mathrm{H}), 7.69(\mathrm{~d}, J=$ $9.0 \mathrm{~Hz}, 1 \mathrm{H}), 7.61-7.49(\mathrm{~m}, 6 \mathrm{H}), 7.30(\mathrm{dd}, J=6.8,2.0 \mathrm{~Hz}, 1 \mathrm{H}), 6.91(\mathrm{~d}, J=9.0 \mathrm{~Hz}, 1 \mathrm{H}), 6.1$ (enol s, 0.92H). ${ }^{13} \mathrm{C}$ NMR $\delta 184.0,154.0,139.7,137.7,136.1,130.9,130.3,128.2,127.5,126.6$, 123.6, 123.2, 122.2, 118.1, 89.8.

1-Fur-2-yl-2-(pyrid-2-yl)-1-ethanone (10h). Yellow needles (84\%), mp 54-55 ${ }^{\circ} \mathrm{C}$ (lit. $^{22 \mathrm{a}}$ 49.5-51.0 $\left.{ }^{0} \mathrm{C}\right) .{ }^{1} \mathrm{H}$ NMR. [keto + enol] $\delta 8.55(\mathrm{~d}, J=4.4 \mathrm{~Hz}, 1 \mathrm{H}), 8.18(\mathrm{~d}, J=5.1 \mathrm{~Hz}, 1 \mathrm{H})$, $7.67-7.55(\mathrm{~m}, 3 \mathrm{H}), 7.46(\mathrm{~s}, 1 \mathrm{H}), 7.35-7.31(\mathrm{~m}, 2 \mathrm{H}), 7.19-7.15(\mathrm{~m}, 1 \mathrm{H}), 7.03(\mathrm{~d}, J=8.2 \mathrm{~Hz}, 1 \mathrm{H})$, $6.92(\mathrm{t}, J=6.0 \mathrm{~Hz}, 1 \mathrm{H}), 6.84(\mathrm{~d}, J=3.3 \mathrm{~Hz}, 1 \mathrm{H}), 6.54$ (br s, 1H), 6.49 (br s, 1H), 6.03 (enol s, $0.32 \mathrm{H}$ ), 4.34 (keto s, 1.36H). ${ }^{13} \mathrm{C}$ NMR [keto + enol] $\delta 185.4,169.9,152.3,151.3,149.5$ (2C), 146.8 (2C), 143.0, 137.2, 136.5, 132.3, 124.1, 122.0, 121.6, 118.5, 117.7, 112.4, 111.7, 109.4, 92.4, 48.0. Anal. Calcd. For $\mathrm{C}_{11} \mathrm{H}_{9} \mathrm{NO}_{2}$ : C, 70.58; H, 4.85; N, 7.48. Found: C, 70.51; H, 4.91; N, 7.36 .

2-(Pyrid-2-yl)-1-(thien-2-yl)-1-ethanone (10i). Pale yellow oil ${ }^{22 a}$ (68\%). ${ }^{1} \mathrm{H}$ NMR [keto + enol] $\delta 8.58(\mathrm{ddd}, J=4.9,1.8,0.8 \mathrm{~Hz}, 1 \mathrm{H}), 8.06(\mathrm{ddd}, J=4.9,1.7,0.9 \mathrm{~Hz}, 1 \mathrm{H}), 7.87$ (dd, $J=3.9,1.3$ $\mathrm{Hz}, 1 \mathrm{H}), 7.66(\mathrm{td}, J=7.6,1.8 \mathrm{~Hz}, 1 \mathrm{H}), 7.63(\mathrm{dd}, J=4.9,1.2 \mathrm{~Hz}, 1 \mathrm{H}), 7.57(\mathrm{~d}, J=1.8 \mathrm{~Hz}, 1 \mathrm{H})$, $7.55(\mathrm{dd}, J=2.9,1.1 \mathrm{~Hz}, 1 \mathrm{H}), 7.52(\mathrm{dd}, J=3.9,1.3 \mathrm{~Hz}, 1 \mathrm{H}), 7.34-7.43(\mathrm{~m}, 1 \mathrm{H}), 7.19$ (ddd, $J=$ $8.9,5.1,1.2 \mathrm{~Hz}, 1 \mathrm{H}), 7.11(\mathrm{dd}, J=5.1,3.9 \mathrm{~Hz}, 1 \mathrm{H}), 7.05(\mathrm{dd}, J=5.1,3.6 \mathrm{~Hz}, 1 \mathrm{H}), 6.98(\mathrm{dt}, J=$ 8.4, $0.9 \mathrm{~Hz}, 1 \mathrm{H}), 6.84$ (ddd, $J=7.2,5.4,1.2 \mathrm{~Hz}, 1 \mathrm{H}), 5.95$ (enol s, $0.15 \mathrm{H}), 4.45$ (keto s, 1.7H). ${ }^{13} \mathrm{C}$ NMR [keto+enol] $\delta 189.6,157.5,154.7,149.4,143.7,141.3,137.4,136.7,134.3,133.4$, $128.2,127.6,127.1,125.4,125.3,124.2,122.1,121.4,116.7,115.0,91.5,48.9$.

2-(Pyrid-2-yl)-1-(pyrid-3-yl)-1-ethanone (10j). Yellow prisms (72\%), mp 70-71 ${ }^{\circ} \mathrm{C}$ (lit. ${ }^{22 b}$ 69-70 $\left.{ }^{\circ} \mathrm{C}\right) .{ }^{1} \mathrm{H}$ NMR $[$ keto + enol] $\delta 15.80(\mathrm{~s}, 1 \mathrm{H}), 9.27(\mathrm{dd}, J=2.4,0.9 \mathrm{~Hz}, 1 \mathrm{H}), 9.07$ (dd, $J=$ 2.4, $0.9 \mathrm{~Hz}, 1 \mathrm{H}), 8.76(\mathrm{dd}, J=4.8,1.5 \mathrm{~Hz}, 1 \mathrm{H}), 8.60(\mathrm{dd}, J=4.8,1.5 \mathrm{~Hz}, 1 \mathrm{H}), 8.34$ (dt, $J=9.9$, $2.4 \mathrm{~Hz}, 1 \mathrm{H}), 8.29$ (d, $J=5.1 \mathrm{~Hz}, 1 \mathrm{H}), 8.11(\mathrm{dt}, J=8.4,1.8 \mathrm{~Hz}, 1 \mathrm{H}), 7.67$ (d, $J=1.8 \mathrm{~Hz}, 1 \mathrm{H})$, 7.64 (dd, $J=1.8,0.9 \mathrm{~Hz}, 1 \mathrm{H}), 7.62$ (d, $J=1.5 \mathrm{~Hz}, 1 \mathrm{H}), 7.40$ (ddd, $J=7.8,4.8,0.9 \mathrm{~Hz}, 1 \mathrm{H}), 7.34$ (ddd, $J=7.8,4.8,0.9 \mathrm{~Hz}, 1 \mathrm{H}), 7.30$ (br s, 1H), 7.19 (ddd, $J=7.5,5.4,1.2 \mathrm{~Hz}, 1 \mathrm{H}), 7.10$ (dd, $J=$ 
8.1, $0.9 \mathrm{~Hz}, 1 \mathrm{H}$ ), 7.02 (ddd, $J=7.5,5.4,1.2 \mathrm{~Hz}, 1 \mathrm{H}$ ), 6.09 (enol s, 0.84H), 4.50 (keto s, 0.32H).

${ }^{13} \mathrm{C}$ NMR [keto + enol] $\delta 195.6,162.3,158.0,154.4,153.5,150.0,150.0,149.7,147.1,143.9$, 137.4, 136.6, 136.0, 132.8, 132.2, 131.8, 124.1, 123.5, 123.1, 122.1, 121.7, 118.9, 94.8, 48.6.

1-(Fur-2-yl)-2-(pyrimidin-4-yl)-1-ethanone (10k). Yellow prisms (50\%), mp128-130 ${ }^{\circ} \mathrm{C} .{ }^{1} \mathrm{H}$ NMR [keto + enol] $\delta 9.16(\mathrm{~d}, J=1.2 \mathrm{~Hz}, 1 \mathrm{H}), 8.70(\mathrm{~s}, 1 \mathrm{H}), 8.68(\mathrm{~s}, 1 \mathrm{H}), 8.31(\mathrm{~d}, J=5.7 \mathrm{~Hz}$, $1 \mathrm{H}), 7.63(\mathrm{dd}, J=1.8,0.7 \mathrm{~Hz}, 1 \mathrm{H}), 7.51$ (br s, 1H), 7.39 (dd, $J=5.1,1.5 \mathrm{~Hz}, 1 \mathrm{H}), 7.34(\mathrm{~d}, J=$ $3.6 \mathrm{~Hz}, 1 \mathrm{H}), 6.96$ (br d, $J=3.3 \mathrm{~Hz}, 1 \mathrm{H}), 6.86(\mathrm{br} \mathrm{d}, J=5.7 \mathrm{~Hz}, 1 \mathrm{H}), 6.58(\mathrm{dd}, J=3.6,1.8 \mathrm{~Hz}$, 1H), 6.50-6.53 (m, 1H), 5.97 (enol s, 0.5H), 4.33 (keto s, 1H). ${ }^{13} \mathrm{C}$ NMR [ [keto + enol] $\delta 183.7$, 163.4, 163.0, 161.4, 158.8, 156.9, 154.7, 153.1, 150.6, 147.1, 144.3, 121.8, 118.7, 117.1, 112.6, 112.0 (2C), 112.0, 91.0, 47.1. Anal. Calcd. For $\mathrm{C}_{10} \mathrm{H}_{8} \mathrm{~N}_{2} \mathrm{O}_{2}: \mathrm{C}, 63.82 ; \mathrm{H}, 4.28 ; \mathrm{N}, 14.89$. Found: C, 63.81; H, 4.22; N, 14.85 .

2-(Quinolin-4-yl)-1-(thien-2-yl)-1-ethanone (10l). Yellow prisms (66\%), mp 66-68 ${ }^{\circ} \mathrm{C} .{ }^{1} \mathrm{H}$ NMR $\delta 8.34(\mathrm{~d}, J=4.5 \mathrm{~Hz}, 1 \mathrm{H}), 8.12(\mathrm{dd}, J=7.9,0.7 \mathrm{~Hz}, 1 \mathrm{H}), 7.94(\mathrm{dd}, J=8.5,0.9 \mathrm{~Hz}, 1 \mathrm{H})$, 7.84 (dd, $J=3.9,0.9 \mathrm{~Hz}, 1 \mathrm{H}), 7.73-7.67$ (m, 2H), 7.54 (ddd, $J=8.2,6.9,1.3 \mathrm{~Hz}, 1 \mathrm{H}), 7.30$ (d, $J$ $=4.5 \mathrm{~Hz}, 1 \mathrm{H}), 7.14(\mathrm{dd}, J=4.9,3.9 \mathrm{~Hz}, 1 \mathrm{H}), 4.64(\mathrm{~s}, 2 \mathrm{H}) .{ }^{13} \mathrm{C}$ NMR $\delta 188.6,150.0,148.4$, 143.3, 140.5, 134.5, 132.6, 130.2, 129.3, 128.3, 127.6, 126.8, 123.6, 122.7, 42.8. Anal. Calcd. For $\mathrm{C}_{15} \mathrm{H}_{11} \mathrm{NOS}$ : C, 71.12; H, 4.38; N, 5.53. Found: C, 70.73; H, 4.29; N, 5.42.

1-(4-Chlorophenyl)-2-phenyl-2-(pyrid-4-yl)-1-ethanone (10m). Oil (63\%). ${ }^{1} \mathrm{H}$ NMR $\delta 8.50$ $(\mathrm{dd}, J=6.1 \mathrm{~Hz}, 2 \mathrm{H}), 7.90(\mathrm{dd}, J=8.7 \mathrm{~Hz}, 2 \mathrm{H}), 7.36(\mathrm{dd}, J=8.7 \mathrm{~Hz}, 2 \mathrm{H}), 7.25-7.33(\mathrm{~m}, 5 \mathrm{H})$, $7.14(\mathrm{dd}, J=6.1 \mathrm{~Hz}, 2 \mathrm{H}), 5.94(\mathrm{~s}, 1 \mathrm{H}) .{ }^{13} \mathrm{C} \mathrm{NMR} \delta 195.4,149.7,147.7,139.8,136.7,134.3$, 130.2, 129.2, 128.9, 128.8, 127.8, 124.2, 58.5. Anal. Calcd. For $\mathrm{C}_{19} \mathrm{H}_{14} \mathrm{ClNO}$ : N, 4.55. Found: $\mathrm{N}$, 4.61.

1-[4-(Diethylamino)phenyl]-2-phenyl-2-(pyrid-4-yl)-1-ethanone (10n). Colorless prisms (67\%), mp 134-136 ${ }^{\circ} \mathrm{C} .{ }^{1} \mathrm{H}$ NMR $\delta 8.50(\mathrm{dd}, J=6.1 \mathrm{~Hz}, 2 \mathrm{H}), 7.89$ (d, $\left.J=9.2 \mathrm{~Hz}, 2 \mathrm{H}\right), 7.24-7.33$ $(\mathrm{m}, 6 \mathrm{H}), 7.21(\mathrm{dd}, J=6.1 \mathrm{~Hz}, 2 \mathrm{H}), 6.57(\mathrm{~d}, J=9.2 \mathrm{~Hz}, 2 \mathrm{H}), 3.36(\mathrm{q}, J=7.1 \mathrm{~Hz}, 4 \mathrm{H}), 1.16(\mathrm{t}, J=$ $7.1 \mathrm{~Hz}, 6 \mathrm{H}) .{ }^{13} \mathrm{C}$ NMR $\delta 193.9,151.3,149.7,149.0,138.2,131.5,128.9,128.8,128.7,127.3$, 124.6, 110.2, 57.5, 44.4, 12.4. Anal. Calcd For $\mathrm{C}_{23} \mathrm{H}_{24} \mathrm{~N}_{2} \mathrm{O}: \mathrm{C}, 80.20 ; \mathrm{H}, 7.02 ; \mathrm{N}, 8.13$. Found: $\mathrm{C}$, $80.54 ; \mathrm{H}, 7.26 ; \mathrm{N}, 8.25$.

1-(4-Chlorophenyl)-2-(quinolin-4-yl)-1-ethanone (10o). Colorless plates (87\%), mp 138-139 ${ }^{\circ} \mathrm{C} .{ }^{1} \mathrm{H}$ NMR $\delta 8.84(\mathrm{~d}, J=4.3 \mathrm{~Hz}, 1 \mathrm{H}), 8.14(\mathrm{~d}, J=8.4 \mathrm{~Hz}, 1 \mathrm{H}), 7.97(\mathrm{~d}, J=8.5 \mathrm{~Hz}, 2 \mathrm{H}), 7.82$ (d, $J=8.2 \mathrm{~Hz}, 1 \mathrm{H}), 7.70$ (t, $J=7.1,1 \mathrm{H}), 7.53(\mathrm{t}, J=7.1 \mathrm{~Hz}, 1 \mathrm{H}), 7.45(\mathrm{~d}, J=8.5 \mathrm{~Hz}, 2 \mathrm{H}), 7.23$ $(\mathrm{d}, J=4.3 \mathrm{~Hz}, 1 \mathrm{H}), 4.67$ (s, 2H). ${ }^{13} \mathrm{C}$ NMR $\delta$ 194.7, 150.0, 148.3, 140.6, 140.1, 134.4, 130.3, 129.7, 129.3, 129.1, 127.5, 126.8, 123.4, 122.7, 42.0. Anal. Calcd. For $\mathrm{C}_{17} \mathrm{H}_{12} \mathrm{ClNO}$ : C, 72.47; H, 4.29; N, 4.97. Found: C, 72.30; H, 4.34; N, 4.87.

1-(4-Nitrophenyl)-2-(quinolin-4-yl)-1-ethanone (10p). Pink prisms (72\%), mp $127-128{ }^{\circ} \mathrm{C} .{ }^{1} \mathrm{H}$ NMR $\delta 8.88(\mathrm{~d}, J=4.3 \mathrm{~Hz}, 1 \mathrm{H}), 8.35(\mathrm{~d}, J=8.1 \mathrm{~Hz}, 2 \mathrm{H}), 8.22(\mathrm{~d}, J=8.1 \mathrm{~Hz}, 2 \mathrm{H}), 8.16(\mathrm{~s}, 1 \mathrm{H})$, $7.83(\mathrm{~d}, J=8.1 \mathrm{~Hz}, 1 \mathrm{H}), 7.75(\mathrm{t}, J=8.1 \mathrm{~Hz}, 1 \mathrm{H}), 7.58(\mathrm{t}, J=7.6 \mathrm{~Hz}, 1 \mathrm{H}), 7.28(\mathrm{~d}, J=4.3 \mathrm{~Hz}$, 1H), 4.79 (s, 2H). ${ }^{13} \mathrm{C}$ NMR $\delta 194.0,163.0,150.0,140.6,139.9,130.5,129.6,129.5,127.2$, 124.1, 123.3, 122.8, 113.1, 42.7. Anal. Calcd. For $\mathrm{C}_{17} \mathrm{H}_{12} \mathrm{~N}_{2} \mathrm{O}_{3}: \mathrm{N}, 9.58$. Found: $\mathrm{N}, 9.43$. 


\section{Acknowledgements}

We thank Dr. Suman Majumder who prepared of 2-(2-pyridinyl)-1-(3-pyridinyl)-1-ethanone and 1-(4-nitrophenyl)-2-(4-quinolinyl)-1-ethanone.

\section{References}

1. (a) Revesz, L.; Padova, F. E. D.; Buhl, T.; Feifel, R.; Gram, H.; Hiestand, P.; Manning, U.; Zimmerlin, A. Bioorganic \& Med. Chem. Lett. 2000, 10, 1261. (b) Olivera, R.; SanMartin, R.; Dominguez, E.; Solans, X.; Urtiaga, M. K.; Arriortua, M. I. J. Org. Chem. 2000, 65, 6398. (c) Chen, C.; Zhu, Y.-F.; Liu, X.-J.; Lu, Z.-X.; Xie, Q.; Ling, N. J. Med. Chem. 2001, 44, 4001. (d) Churruca, F.; SanMartin, R.; Tellitu, I.; Dominguez, E. Org. Lett. 2002, 1591. (e) Veeramaneni, V. R.; Pal, M.; Yeleswarapu, R. Tetrahedron 2003, 59, 3283. (f) Mamolo, M. G.; Zampieri, D.; Falagiani, V. ARKIVOC 2004, (xi), 231.

2. (a) El-Dissouky, A.; Masoud, M. S. Transition Metal. Chemistry 1984, 9, 327. (b) Adu Zuhri, A. Z.; El-Dissouky, A. Mikrochim. Acta 1991, 111.

3. Chesunt, R. W.; Cesati III, R. R.; Cutler, C. S.; Pluth, S. L.; Katzenellenbogen, J. A. Organometallics 1998, 17, 4889.

4. Ohtsuka, Y.; Ikeno, T.; Yamada, T. Tetrahedron: Asymmetry 2000, 11, 3671.

5. Baker, B. R.; McEvoy, F. J. J. Org. Chem. 1955, 20, 118.

6. Ishibashi, F.; Tanabe, S.; Oda, T.; Iwao, M. J. Nat. Chem. 2002, 65, 500.

7. Yu, C.-Y.; Meth-Cohn, O. Tetrahedron Lett. 1999, 40, 6665.

8. Terry Jr.; A. V.; Williamson, R.; Gattu, M.; Beach, J. W.; McCurdy, C. R.; Sparks, J. A.; Pauly, J. R. Neuropharmacology 1998, 37, 93.

9. Cèlanire, S.; Salliot-Maire, I.; Ribèreau, P.; Godard, A.; Quèguiner, G. Tetrahedron 1999, 55, 9269.

10. (a) Reddy, M. V. R.; Faulkner, D. J.; Venkateswarlu, Y.; Rao, M. R. Tetrahedron 1997, 53, 3457. (b) Strunz, G. M.; Findlay, J. A. Pyridine and Piperidine Alkaloids. In The Alkaloids; Brossi, A., Ed.; Academic Press: New York, 1985; Vol. 26, pp 89-174. (c) Guarna, A.; Belle, C.; Machetti, F.; Occhiato, E. G.; Cassiani, C.; Comerci, A.; Danza, G.; De Bellis, A.; Dini, S.; Marrucci, A.; Serio, M. J. Med. Chem. 1997, 40, 1112.

11. Rochford, J.; Sen, A. P.; Quirion, R. J. Pharmsc. Exp. Ther. 1996, 277, 1267.

12. Goodman, Gilman, A.; Rall, T. W.; Nies, A. S.; Taylor, P. The Pharmacological Basis of Therapeutics, $8^{\text {th }}$ Ed.; Pergamon: New York, 1990; pp 166.

13. Hewitt, L. E.; Wade, D. R.; Sinsheimer, J. E.; Wang, J. H.; Drach, J. C.; Burckhalter, J. H. J. Med. Chem. 1978, 21, 1339.

14. (a) Heiner, G. H.; Alfons, E. B.; Werner, K.; Karl-Heinz, B. W. EP869121, 1998; Chem. Abstr. 1998, 129, 302559. (b) Kirsch, R. B.; Enhsen, A. B.; Glombik, H.; Kramer, W. M.-L.; 
Eugen, F. WO 0020393, 2000; Chem. Abstr. 2000, 132, 279546. (c) Renga, J. M.; McLaren, K. L.; Ricks, M. J. Organic Process Research \& Development 2003, 7, 267.

15. Lesac, A.; Moslavac-Forjan, D.; Bruce, D. W.; Sunjic, V. Helv. Chim. Acta 1999, 82, 1707.

16. Nishiwaki, N.; Minakata, S.; Komatsu, M.; Ohshiro, Y. Synlett 1990, 273.

17. Nicola, T.; Vieser, R.; Eberbach, W. Eur. J. Org. Chem. 2000, 527.

18. (a) Hay, J. V.; Hudlicky, T.; Wolfe, J. F. J. Am. Chem. Soc. 1975, 97, 5374. (b) Komin, A.P.; Wolfe, J. F. J. Org. Chem. 1977, 42, 2481. (c) Nazareno, M. A.; Rossi, R. A. Tetrahedron Lett. 1994, 35, 185.

19. Yu, C.-Y.; Tayler, D. L.; Meth-Cohn, O. Tetrahedron Lett. 1999, 40, 6661.

20. Reichardt, C.; Che, D.; Heckenkemper, G.; Schafer, G. Eur. J. Org. Chem. 2001, 2343.

21. Khutova, B. M.; Klyuchko, S. V.; Prikazchikova, L. P.; Cherkasov, V. M. J. Heterocycl. Chem. 1982, 522.

22. (a) Goldberg, N. N.; Barkley, L. B.; Levine, R. J. Am. Chem. Soc. 1951, 73, 4301. (b) Goldberg, N. N.; Levine, R. J. Am. Chem. Soc. 1952, 74, 5217. (c) Levine, R.; Raynolds, S. J. Org. Chem. 1960, 25, 530. (d) Sund, E. H.; Lowe, W. D. J. Chem. Eng. Data 1983, 28, 137. (e) Sund, E. H.; Strickland, S. K. J. Chem. Eng. Data 1988, 33, 216.

23. Cassity, R. P.; Tayler, L. T.; Walfe, J. F. J. Org. Chem. 1978, 43, 2286.

24. (a) Katritzky, A. R.; He, H.-Y.; Suzuki, K. J. Org. Chem. 2000, 65, 8210. (b) Katritzky, A. R.; Yang, H.; Zhang, S.; Wang, M. ARKIVOC 2002, (xi), 39.

25. Katritzky, A. R.; Denisko, O. V.; Fang, Y.; Zhang, L.; Wang, Z. ARKIVOC 2001, (xi), 41.

26. Katritzky, A. R.; Abdel-Fattah, A. A. A.; Wang, M. J. Org. Chem. 2003, 68, 1443.

27. Katritzky, A. R.; Abdel-Fattah, A. A. A.; Wang, M. J. Org. Chem. 2003, 68, 4932.

28. Katritzky, A. R.; Pastor, A. J. Org. Chem. 2000, 65, 3679.

29. Gore, T. L.; Rogers, H. N., Jr.; Schumacher, R. M.; Sund, E. H.; Weaver, T. J. J. Chem. Eng. Data 1971, 16, 491.

30. Kolehmainen, E.; Osmialowski, B.; Krygowski, T. M.; Kauppinen, R.; Nissinen, M.; Gawinecki, R. J. Chem. Soc. Perkin Trans. 2 2000, 1259.

31. Kolehmainen, E.; Osmialowski, B.; Nissinen, M.; Kauppinen, R.; Gawinecki, R. J. Chem. Soc. Perkin Trans. 2 2000, 2185. 